\title{
Productivity improvement of an automobile inspection station
}

\begin{abstract}
Vehicle inspection is a procedure mandated by national or a sub national government in many countries, in which a vehicle is inspected to ensure that it conforms to regulations governing safety, emissions, or both. Inspection can be required at various times, e.g., periodically or on the transfer of title to a vehicle. If required periodically, it is often termed periodic motor vehicle inspection; typical intervals are every two years and every year. This project was to find out possible solutions and alternatives in order to achieve an increase in the efficiency and effectiveness of an automobile inspection station. The main problem was to find out the inspection point in the station consuming largest amount of time which causes increase of flow time in the inspection lanes. This causes an increase in the queue length and decrease in customer satisfaction also increases the cost of inspection. Using the tools from motion and time study and software ARENA we simulated the system and predict the changes expected to occur in the inspection lanes. Arena was discrete event simulation and automation software developed by Rockwell Automation in 2000. It uses the SIMAN processor and simulation language, which lead to predict solutions which can reduce the flow time in inspection lanes which can yield an expected improvement in the production capacity.
\end{abstract}

Keywords: productivity, standard time rating, motion study, time study, arena simulation

\author{
Volume 4 Issue 3 - 2018
}

\section{Basil Joy, Shunmugesh K, Pramod Kumar M \\ Department of Mechanical Engineering,Viswajyothi College of Engineering and Technology, India}

Correspondence: Basil Joy, Department of Mechanical Engineering,Viswajyothi College of Engineering and Technology, India,Tel 996191।703, Email basil.joy0009@gmail.com

Received: May 15, 2018 | Published: June 26, 2018

\section{Introduction}

The design of the method of performing an operation when a new product is being put into production, or the improvement of a method already in effect, is a very important part of motion and time study. The logical and systematic approach to solve almost any problem includes

a. Problem definition and analysis;

b. Search for possible solutions;

c. Evaluation and discussion the alternatives and

d. Recommendation for action. Here we have taken the example of a vehicle inspection station.

The aim of carrying out this project is to improve and enhance the productivity of the inspection station. Here, we find out total inspection time required for the process and find out methods to reduce it. There are several ways of reducing the total time. For example we can find out the inspection elements in the process which consume more time and increase the flow time in the inspection lanes. Then we can find ways to reduce time for these elements. This research investigated and searched for possible solutions and alternatives aimed at achieving the objective using some tools from motion and time study and ARENA software to simulate and predict the changes expected to occur in the inspection lanes. Using the above results, we can to increase the efficiency of inspection centre by increasing the productivity. This can also lead to increase in profit as well as customer satisfaction.

\section{Simulation with arena}

Arena is discrete event simulation and automation software developed by Rockwell Automation in 2000.

It uses the SIMAN processor and simulation language. As of June 2012, it is in version 14 (first version with online 3D visualization tool).

\section{Description of the present system}

\section{Inspection stages}

Inspection stages involved in the station are given as following. All the main elements in the inspection stages of the process are also written along with it.

First stage (exterior body): Confirmation of actual vehicle identity against the legal documents (noting down the chassis number), Visual check of the vehicle covering 40 items such as wheels, lights, glasses, and driving wheel. All results are directly entered to the computer. Road test for the vehicle to check for door, dashboard and bumper noise, checking clutch and gear operation, steering and suspension noise and any other abnormal noise.

Second stage: Engine room check for checking engine oil level, battery water level, Coolant level, Brake fluid level, Wiper bottle water level.

Third stage: Fully automated check for side slip of front wheels using sensors.

Fourth stage: Check for head lamps using a dashboard.

Fifth stage: Exhaust test to check emission levels of CO (carbon monoxide)

Sixth stage: Under carriage test for checking leakage of silencer and steering system, checking of lower arms, loose nuts and bolts.

Seventh stage: Overall inspection of whole vehicle is done by the final inspector.

\section{Problems in present system}

The problems observed in the inspection process were,

1. Identification of the inspection points which consumes more time causing the increase of flow time which increases the queue 
length and decreases the customer satisfaction. (Queue formed at service station 1, station 3, \&station 9 for visual check and road test. Queue formed for wheel alignment test at station 6.Queue formed at final inspection)

a. There is insufficient number of inspectors at some stages.

b. There are no separate lanes for newly arrived vehicles and failed vehicles.

c. Certain inspection elements are repeated during subsequent stages.

Methodology used for solving these problems uses the principle of motion and time study. Using work methods design we have

a. Elimination of all unnecessary work

b. Combine operations or element.

c. Change sequence of operations

\section{Methodology}

Generally three types of service are being done at the service centre. These are described as follows,

\section{Formulate - problem}

a. To simulate the present condition of the service system at the service centre

b. To find number of vehicles in the queue \& average waiting time of vehicles

\section{Collect the data $\&$ define the model}

Collect the following data:

a. Number of vehicles arriving at the service station

b. The time between the arrivals of two vehicles i.e., inter arrival time of vehicles

c. Service times for respective processes at each station

\section{Define the model}

The arrival process can be modeled with the help of create module with the parameter as inter arrival time in GAMMA distribution with expression GAMMA $(3.9,4.6)$ in minutes.

a. We have ten service stations in total at seven service points respectively.

b. There are 3 individual stations for visual checking for paid, free and accident service cases.

c. There are 2 individual stations for engine check for paid and free service cases.

d. Based on data collected we have fixed that out of the total vehicles arriving

e. $22 \%$ are coming for free service, $48 \%$ for paid service, $30 \%$ are coming for accident service.

f. Based on type of service required, vehicles are sending to respective stations.

\section{Model building}

Modeling, including simulation modeling, is a complicated activity that combines art and science. The major steps are listed below.

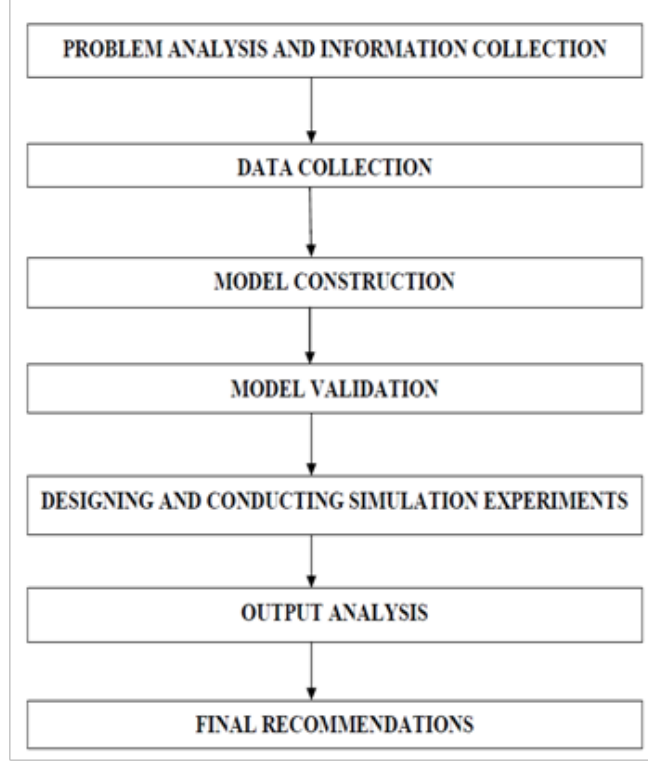

Figure I simulation modeling.

Problem analysis and information collection: The first step in building a simulation model is to analyze the problem itself. Note that system modelling is rarely undertaken for its own sake. Rather, modelling is prompted by some system oriented problem whose solution is the mission of the underlying project. In order to facilitate a solution, the analyst first gathers structural information that bears on the problem, and represents it conveniently.

Data collection: Data collection is needed for estimating model input parameters. The analyst can formulate assumptions on the distributions of random variables in the model. When data are lacking, it may still be possible to designate parameter ranges, and simulate the model for all or some input parameters in those ranges. Data collection is also needed for model validation. That is, data collected on system output statistics are compared to their model counterparts (predictions).

Model construction: Once the problem is fully studied and the requisite data collected, the analyst can proceed to construct a model and implement it as a computer program. The computer language employed may be a general-purpose language (e.g., C++, Visual Basic, FORTRAN) or a special-purpose simulation language or environment (e.g., Arena, Pro-model, GPSS).

Model verification: The purpose of model verification is to make sure that the model is correctly constructed. Differently stated, verification makes sure that the model conforms to its specification and does what it is supposed to do.

Model validation: Every model should be initially viewed as a mere proposal, subject to validation. Model validation examines the fit of the model to empirical data (measurements of the real-life system to be modelled).

Designing and conducting simulation experiments: Once the analyst judges a model to be valid, he or she may proceed to design a 
set of simulation experiments (runs) to estimate model performance and aid in solving the project's problem (often the problem is making system design decisions). The analyst selects a number of scenarios and runs the simulation to glean insights into its workings.

Output analysis: The estimated performance measures are subjected to a thorough logical and statistical analysis. A typical problem is one of identifying the best design among a number of competing alternatives. A statistical analysis would run statistical inference tests to determine whether one of the alternative designs enjoys superior performance measures, and so should be selected as the apparent best design

Final recommendations: Finally, the analyst uses the output analysis to formulate the final recommendations for the underlying systems problem. This is usually part of a written report. ${ }^{1-5}$

\section{Conclusion}

\section{Possible solutions or alternatives}

Here we have developed possible solutions or alternatives which can be used to reduce the total inspection time. These solutions can be extremely useful in order to increase the productivity of the system in the long run. The following steps or methods have been developed:

a. Use bar code reader to read the chassis number instead of manual reading: The reading and noting down of chassis number consumes a lot of time. So, a bar code reader can be useful so as to reduce the time. About $2.7 \%$ of the total time is reduced by using a bar code reader by real experiments

b. Combine elements in the inspection: We can combine the elements in the inspection like "inspects the lights" and element "inspects the rubbers" in one step. Use of both the hands simultaneously is a methodology not widely used.

c. Division of inspection lanes: In order to minimise the waiting time of vehicles at a station or stage, the inspection lane can be divided into two sections i.e. one for the newly arrived vehicles and another for failed vehicles which have come back again after the final inspection. This set up will be useful to workers as well since it avoids the confusion.

d. Certain operations can be done during some other inspection is being done without disturbing that process. For example, visual check process for the vehicle can be during the head lamps test or exhaust test so that we can save the time. This type of reshuffling can be used so that each inspection stage becomes free soon because it allows the continuous flow of work without increasing the number of vehicles waiting in the queue for the service.

e. Adding of second inspector at inspection stage 1: During the first inspection stage a lot of time is consumed for noting down the chassis number, doing the visual check and then entering the data into the computer. This time can be saved by adding a second inspector for doing either of the work and then drive the vehicle to the next stage of inspection.

f. Combine the inspection stages: We can combine operations like wheel alignment, head lamps test and exhaust test. .Installing mirror permanently in front of this combined stage an inspector can be made free and utilised for other stages. In proposed system this inspector is added to visual check in the initial stage.

g. Avoid unnecessary and repetitive checks: We can try to avoid certain checks during inspection which are repetitive.

h. Add multiple stations: Addition of inspection station at each inspection stage can be useful because it increases the capacity of the inspection station. The total inspection time can be reduced and also the waiting time at each station can be minimised.

i. Change the sequence of operations: The sequence of operations can be changed in order to maintain a continuous flow of work and reduce the time for the inspection.

j. Add a helper for final inspection: The final inspection of the vehicle requires the maximum time so that he has to conduct each element in the inspection in a limited period of time. So, by adding a helper we can reduce the total time of final inspection to a certain extent. Almost all the operations or elements can be conducted in half the time with a helper and thus it can be applied. The experiment result shows that overall time is reduced to an average of 7.4 minutes by introducing the inspector. When inspectors are allocated to the first stages and final stages of inspection, the time reduced to half by experimentation. Combination of the three stages (wheel alignment, exhaust test, head lamps test) also reduces $1 / 3$ of the time. In the present system 28 vehicles are inspected per 8 hour. Out of these 10 are fixed for free service which doesn't contribute income.18 of the vehicle contribute income and they are for paid and accident services. By implementing re-arrangements, 37 vehicles can be accommodated per 8 hour. By these 9 vehicles can additionally include and the profit can be maximized. i.e., (9* service cost per vehicle * no of working day per month* 12 ). Thus the productivity leaps.

\section{Acknowledgements}

None.

\section{Conflict of interest}

The author declares there is no conflict of interest.

\section{References}

1. Colin Herron, Paul M Braiden. A Methodology for developing sustainable quantifiable productivity improvement in manufacturing companies. International Journal of Production Economics. 1996;104(1):143-153.

2. McBride P, Bobjer O. Using ergonomics to improve working conditions and reduce costs. Contemporary Ergonomics; 1999:43-47.

3. Shikdar AA, Das B. The relationship between worker satisfaction and productivity in a repetitive industrial task. Appl Ergon. 2003;34(6):603610

4. Towards hypercube queuing models for dispatch policies with priority in queue and partial backup LásaraFabrícia Rodrigues Department of Production Engineering Federal University of OuroPreto, MG, Brazil. 2017.

5. Khalid S Al-Saleh. Productivity improvement of a motor vehicle inspection station using motion and time study techniques. Journal of King Saud University-Engineering Sciences. 2009;23(1):33-41. 